\title{
Hyperbranched polymer drug delivery treatment for lung metastasis of salivary adenoid cystic carcinoma in nude mice
}

\author{
WEIWEI WANG, JIALEI MA, FURONG JIN and JIANXING LIAO
}

\begin{abstract}
Department of Oral and Maxillofacial Surgery, Shanghai Engineering Research Center of Tooth Restoration and Regeneration, School of Stomatology, Tongji University, Shanghai 200072, P.R. China
\end{abstract}

Received January 25, 2017; Accepted July 21, 2017

DOI: $10.3892 /$ etm.2017.4902

\begin{abstract}
Salivary adenoid cystic carcinoma (SACC) is associated with a high rate of lung metastasis. When lung metastasis occurs, the effects of traditional chemotherapy on SACC are poor. Hyperbranched polymer drug delivery (degradable hyperbranched polyglycerols, dHPGs) can be used as a strategy to load several drugs, and obtain beneficial effects on SACC lung metastasis through enhanced permeability and retention. In the present study, hydroxycamptothecin (HPT)-conjugated dHPG (dHPG-HPT) was synthesized and its effects on SACC xenografts in the lungs of nude mice were evaluated. SACC cells with a high potential for pulmonary metastasis (SACC-LM cells) were injected into the tail vein of mice, establishing a nude mouse model. The mice were randomly divided into the three following groups: Control, HPT and dHPG-HPT. Saline (control), HPT or dHPG-HPT were injected into the mice. After two weeks, the mice were euthanized and their lungs were removed. The lungs were paraffin-embedded for hematoxylin and eosin, and immunohistochemical staining analyses. Primary antibodies directed against vascular endothelial growth factor (VEGF), cluster of differentiation 34 (CD34), proliferating cell nuclear antigen (PCNA) and matrix metalloproteinase 9 (MMP9) were used. Gross observation demonstrated that the volumes of SACC lung metastasis nodules were significantly decreased in the dHPG-HPT group compared with the control and HPT groups. Immunohistochemical analysis revealed a lower expression of VEGF, CD34, PCNA and MMP9 in the
\end{abstract}

Correspondence to: Professor Jianxing Liao, Department of Oral and Maxillofacial Surgery, Shanghai Engineering Research Center of Tooth Restoration and Regeneration, School of Stomatology, Tongji University, 399 Middle Yanchang Road, Shanghai 200072, P.R. China

E-mail: ljx@tongji.edu.cn

Abbreviations: SACC, salivary adenoid cystic carcinoma; EPR, enhanced permeability and retention

Key words: EPR effect, hyperbranched polymers, lung metastasis, salivary adenoid cystic carcinoma
dHPG-HPT group. The results of the current study suggest that dHPG-HPT can suppress the growth of SACC xenografts in nude mice, providing a theoretical basis for macromolecular drug delivery-based treatment of SACC.

\section{Introduction}

Salivary adenoid cystic carcinoma (SACC), which is a common malignant tumor of the oral and maxillofacial region, originates from myoepithelial and basal cells, and although this tumor type grows slowly, it has a high capacity for invasion $(1,2)$. SACC easily disseminates to distant organs through the bloodstream, and the most common organ of distant metastases is the lung (3). Clinically, as the main cause of death in patients with SACC, lung metastasis initially occurs as a distant metastasis of SACC and is frequently observed before the first diagnosis. Studies have shown that cumulative lung metastasis rates at 5 and 10 years can be as high as 70 and $100 \%$, respectively. The high lung metastatic characteristics of SACC significantly reduce the long-term survival rate. Studies have reported that the overall survival rate (60 months) of patients with SACC is $71 \%$, whereas the overall survival rate (180 months) is only $37 \%$. However, the 5- and 15-year survival rates of patients diagnosed with lung metastasis are only 35 and $0 \%$, respectively (4). Currently, chemotherapy is the major strategy used to treat lung metastasis. However, traditional chemotherapy drugs have poor solubility and poor targeting, and their use may therefore lead to many side effects and the potential development of drug resistance, which has a significant impact on the effectiveness of chemotherapy.

Recently, new types of hyperbranched polymers have been developed for use as drug delivery systems (5). The use of hyperbranched polymers in the field of biomedicine has only recently emerged (6). As a new drug delivery system, hyperbranched polymers have many excellent properties, including ligand targeting and a large number of terminal functional groups, which can be used to connect drug molecules and fluorescence groups. Different molecular structures can provide a variety of drug delivery pathways. Sensitive responsiveness to environmental stimuli can be used to build an intelligent drug-controlled release system; the relatively small hydrodynamic radius is conducive to the improvement of the efficiency of cell uptake (7). The emergence of hyperbranched polymer 
drug delivery systems provides a good choice for the improvement of the efficacy of chemotherapy for adenoid cystic carcinoma.

Therefore, we used glycerol and glycidyl methacrylate as raw materials to synthesize dHPG. The characterization of dHPG was detected using the nuclear magnetic resonance (NMR) and Fourier transform infrared spectroscopy (FTIR) techniques. An antineoplastic drug, specifically hydroxycamptothecin (HPT), was conjugated onto the surface of dHPG. The drug loading efficiency (DLE) of the resultant HPT-conjugated polymers (dHPG-HPT) was $5.66 \mathrm{wt} \%$ (8). dHPG-HPT was used as a treatment, and the growth of the SACC xenografts in nude mice was observed. Thus, the aim of the present study was to provide new solutions and theoretical bases for clinical chemotherapy.

\section{Materials and methods}

Cell culture. SACC cells with a high potential for pulmonary metastasis (SACC-LM cells) were a gift from the Department of Oral and Maxillofacial Surgery, School of Stomatology, Peking University (Beijing, China). The cells were cultured in DMEM supplemented with $10 \%$ fetal bovine serum and $2 \%$ penicillin/streptomycin at $37^{\circ} \mathrm{C}$ with $5 \% \mathrm{CO}_{2}$.

Xenograft study. Sixteen four-week-old female BALB/c nu/nu nude mice were inoculated with $4 \times 10^{6}$ SACC-LM cells in $200 \mu \mathrm{l}$ of culture medium via the tail vein. Four weeks after inoculation, 4 of the animals were euthanized, and their lungs were removed. Metastatic nodules were observed on the surface of the lungs, which indicated the successful establishment of the animal model of SACC lung metastasis. Subsequently, the remaining animals were randomly divided into the following three groups: Control, HPT and dHPG-HPT group. With regard to treatment, $0.2 \mathrm{ml}$ of $0.9 \%$ saline was injected into the tail vein of nude mice in the control group; $0.2 \mathrm{mg}$ of HPT (Biochempartner Co, Ltd, Shanghai, China) at a concentration of $1 \mu \mathrm{g} / \mathrm{ml}$ was injected into the tail vein of nude mice in the HPT group; and dHPG-HPT (synthesized as described in our previous study) containing the same concentration of HPT was injected into the tail vein of nude mice in the dHPG-HPT group. The treatment was implemented twice a week for two weeks. After two weeks, all mice were euthanized, and their lungs were removed. Finally, the lungs were paraffin-embedded for $\mathrm{H} \& \mathrm{E}$ and immunohistochemical staining analyses. The study protocol was approved by the local Animal Ethics Committee of Tongji University (Shanghai, China) (project no. 2016-009) and was conducted in accordance with the local and state regulations regarding the use of animals for experimental purposes.

$H \& E$ staining. The lungs were immersed in $4 \%$ paraformaldehyde for $4 \mathrm{~h}$ and transferred to $70 \%$ ethanol. The individual lobes of the lung were placed in processing cassettes, dehydrated through a serial alcohol gradient, and embedded in paraffin wax blocks. Prior to staining, 4- $\mu \mathrm{m}$-thick lung tissue sections were deparaffinized in xylene, rehydrated through decreasing concentrations of ethanol, and washed in phosphate-buffered saline (PBS). Subsequently, the tissue sections were stained with $\mathrm{H} \& \mathrm{E}$. After staining, the sections were dehydrated through increasing concentrations of ethanol and xylene.
Immunohistochemical staining. Immunohistochemical studies of the lung tissue were conducted using the following antibodies: Monoclonal rabbit anti-mouse vascular endothelial growth factor (VEGF; ab1316), cluster of differentiation (CD34; ab81289), proliferating cell nuclear antigen (PCNA; ab92552), and MMP9 (ab58803) (dilution 1:400), which were all purchased from Abcam (Cambridge, UK); biotinylated goat anti-rabbit $\operatorname{IgG}$ was used as the secondary antibody. An S-P kit (Gene Tech Biotechnology Co., Ltd., Shanghai, China) was used for the immunohistochemistry experiments. Briefly, the paraffin sections were deparaffinized in xylene and rehydrated in an alcohol gradient. Antigen retrieval was performed by microwave treatment in $0.01 \mathrm{M}$ sodium citrate buffer $(\mathrm{pH}=6.0)$ for $15 \mathrm{~min}$, after which the slides were cooled to room temperature for $1 \mathrm{~h}$, and subsequently washed with PBS. Endogenous peroxidase activity was inactivated with $0.3 \%$ hydrogen peroxide for $30 \mathrm{~min}$ and non-specific protein interactions were blocked with an avidin/biotin blocking solution containing $10 \%$ normal goat serum. The sections were subsequently incubated overnight with primary antibodies against VEGF, CD34, PCNA, and MMP9. The immunoreactions were detected after staining with 3,3'-diaminobenzidine.

Scoring of the immunohistochemistry results. The signal intensities of VEGF and MMP9 were measured using Image-Pro Plus. Briefly, five fields of view were randomly selected, and the integrated optical density (IOD) (sum) and area (sum) of the fields were calculated. The mean optical density was calculated as the IOD (sum)/area (sum).

The nuclei of PCNA-positive cells were stained brown. Five fields of PCNA staining were observed at high magnification under light microscopy, and the number of positive cells was counted. The positive CD34 signal was calculated using Weidner's method (9). The criteria for positive staining included the following:

1) A vascular endothelial cell or endothelial cell cluster, isolated from the peripheral vasculature, tumor cell or other connective tissue identified as a single microvessel.

2) A vessel derived from the same microvascular endothelial cells, which are clear and separated from each other, and can be counted as a separate microvessel.

3) Red blood cells are not necessary to confirm microvessels.

4) Vessels in the sparse region of tumor cells and adjacent benign tissue regions were not counted.

5) Vessels with a luminal diameter of $>10$ erythrocytes were not counted.

The entire section was observed to determine the high blood vessel density and the 'Hot spot' at low magnification, $x 40$. Subsequently, the number of blood vessels stained brown was counted at high magnification, $\mathrm{x} 200$. Two people conducted this procedure using a double-blind method.

Statistical analysis. The statistical analyses were performed using SPSS 20.0. All quantitative data were expressed as the means \pm SEM for each condition. The data were tested for statistical significance using one-way analysis of variance (ANOVA). $\mathrm{P}<0.05$ ( $\mathrm{F}$ values $>\mathrm{F} \alpha$ ) were considered to indicate a statistically significant difference. 

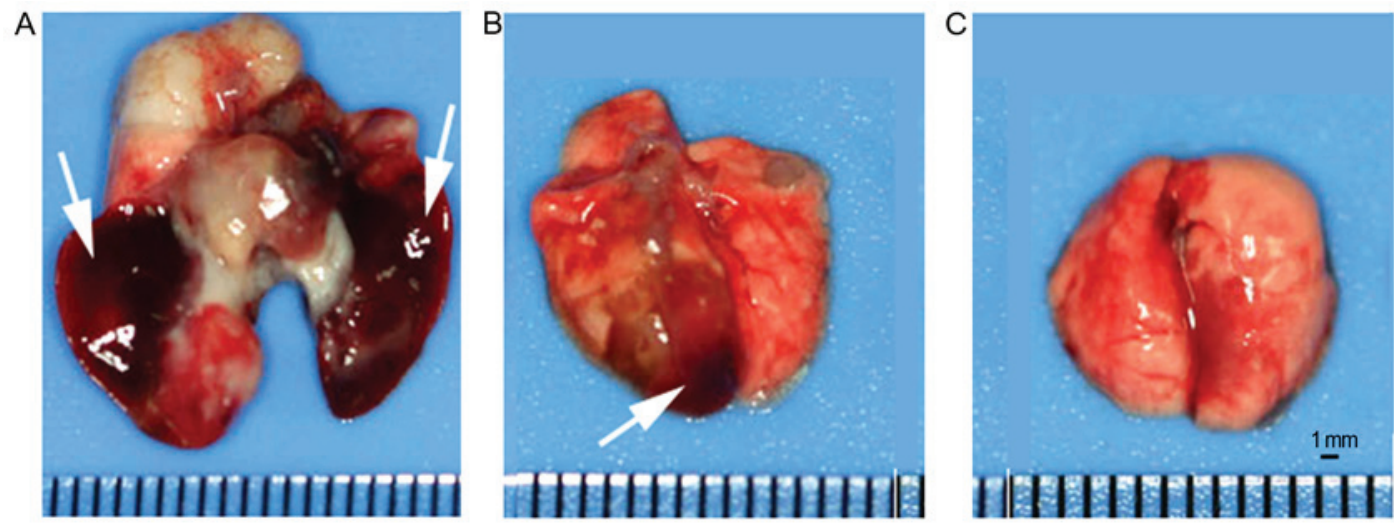

Figure 1. Gross images of metastatic lung tissue. (A) Control group; (B) HPT group; (C) dHPG-HPT group. The arrows indicate the metastatic nodules. Scale bar, $1 \mathrm{~mm}$.
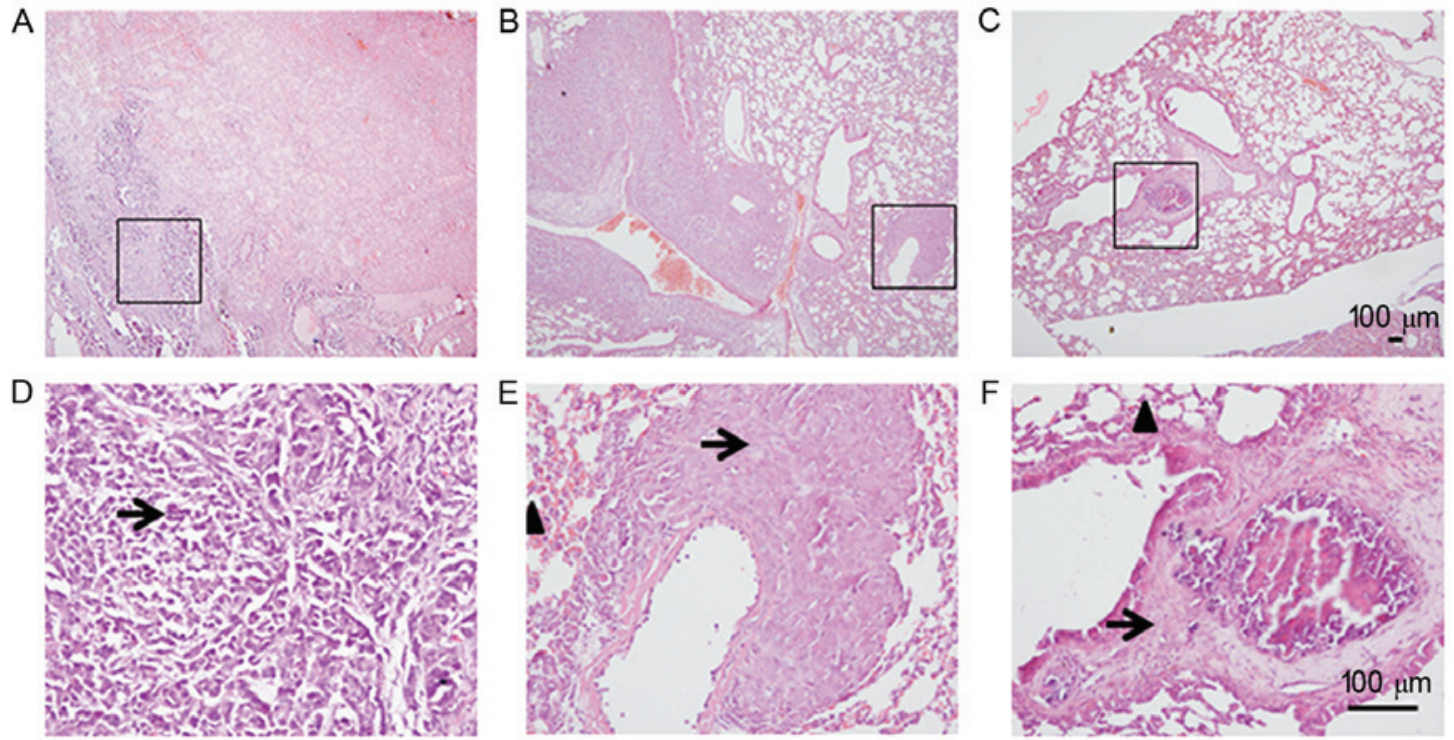

Figure 2. (A-F) Histological aspects of the lungs (H\&E). (A and D) Control group; (B and E) HPT group; (C and F) dHPG-HPT group. The arrows indicate the tumor tissues, and the triangles indicate normal lung tissue. Scale bar, $100 \mu \mathrm{m}$.

\section{Results}

dHPG-HPT inhibits xenograft growth. We initially dissected the lungs of the nude mice in each group and observed that the volumes of the lungs in the control group were the highest (Fig. 1A), the metastatic nodules were large, and the lungs were deformed. Several metastatic nodules were observed on the surface of the lungs of mice in the HPT group (Fig. 1B). The lungs of mice in the dHPG-HPT group were most similar to those of mice in the control group, as few metastatic nodules were observed on the lungs (Fig. 1C).

Next, we observed the histological aspect of the lungs by H\&E staining (Fig. 2). In the control group, a major portion of the lungs was occupied by solid tumor tissue (Fig. 2A). At high magnification, the solid tumor tissue was filled with SACC cells (Fig. 2D). In the HPT group, approximately half of the tissue consisted of solid tumor (Fig. 2B). In contrast to the HPT group, only a few metastatic nodules were observed in the lungs of mice in the dHPG-HPT group (Fig. 2C); a major portion of the tissue was reticular lung tissue.
dHPG-HPT reduces the expression of VEGF and CD34. Angiogenesis plays an important role in the growth of tumors. To explore the molecular differences in angiogenesis in each group, we used immunohistochemistry to detect the expression of VEGF and CD34. Immunohistochemical staining (Fig. 3) of the tumor xenograft showed a marked reduction in the expression of VEGF in the dHPG-HPT group compared with the HPT group [IOD (sum)/area (sum)]: dHPG-HPT, 0.02 vs. HPT, 0.04; P<0.01) (Fig. 3D).

Furthermore, microvessel density (MVD; Fig. 4), which was based on CD34 expression, was significantly lower in the dHPG-HPT group compared with the HPT group (Fig. 4D). This result indicated that dHPG-HPT could reduce angiogenesis in SACC.

dHPG-HPT inhibits the proliferation of SACC cells. We next investigated the inhibitory effect of dHPG-HPT on the proliferation of SACC cells. Immunohistochemistry (Fig. 5) showed that the number of PCNA-positive cells in tumors of the dHPG-HPT group was significantly lower than that 

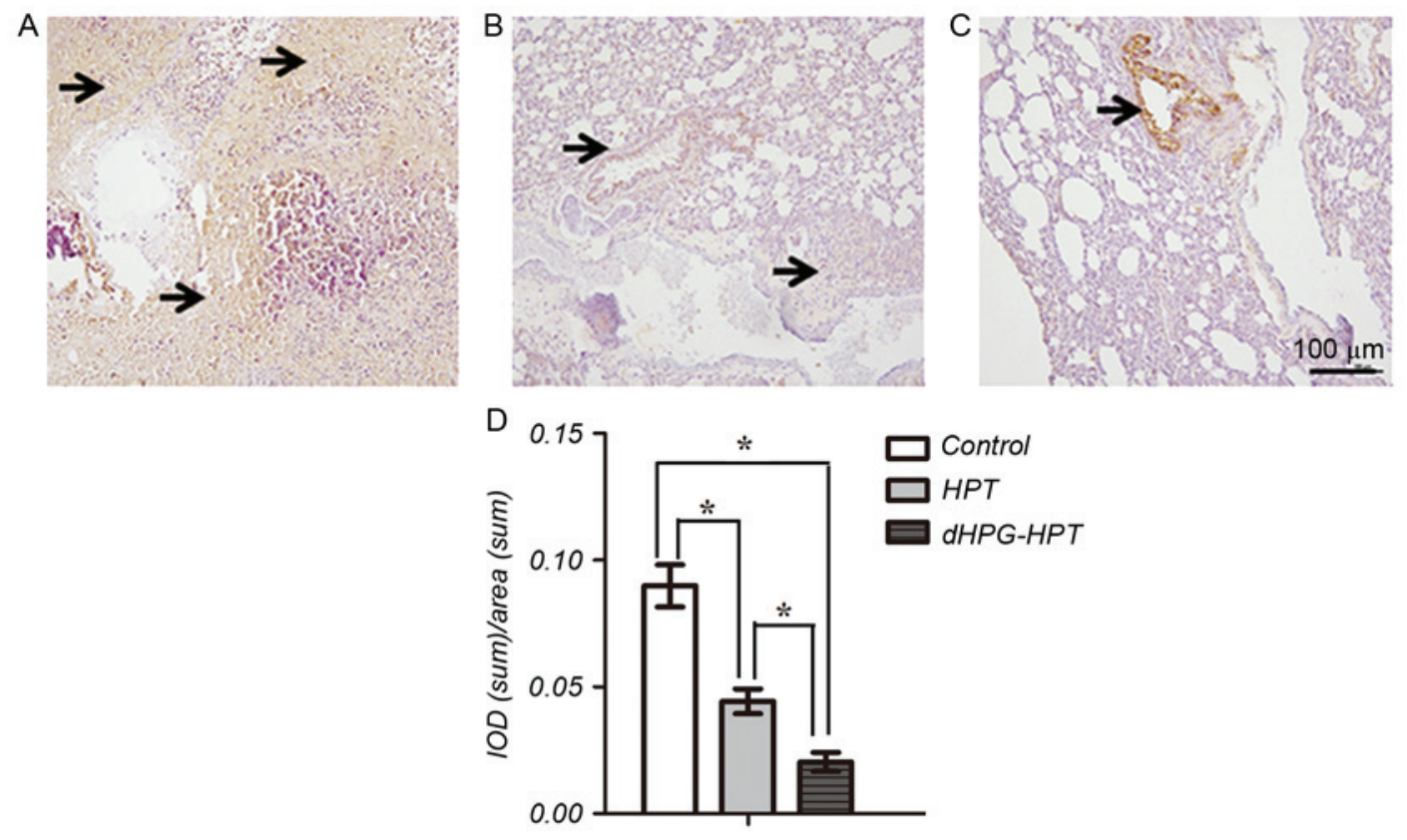

Figure 3. The expression of VEGF in tissue samples from (A) the control group, (B) HPT group and (C) dHPG-HPT group. (D) Shows the IOD (sum)/area (sum) of each group. The arrows indicate the positive signal. Scale bar, $100 \mu \mathrm{m}$. ${ }^{*} \mathrm{P}<0.05$.
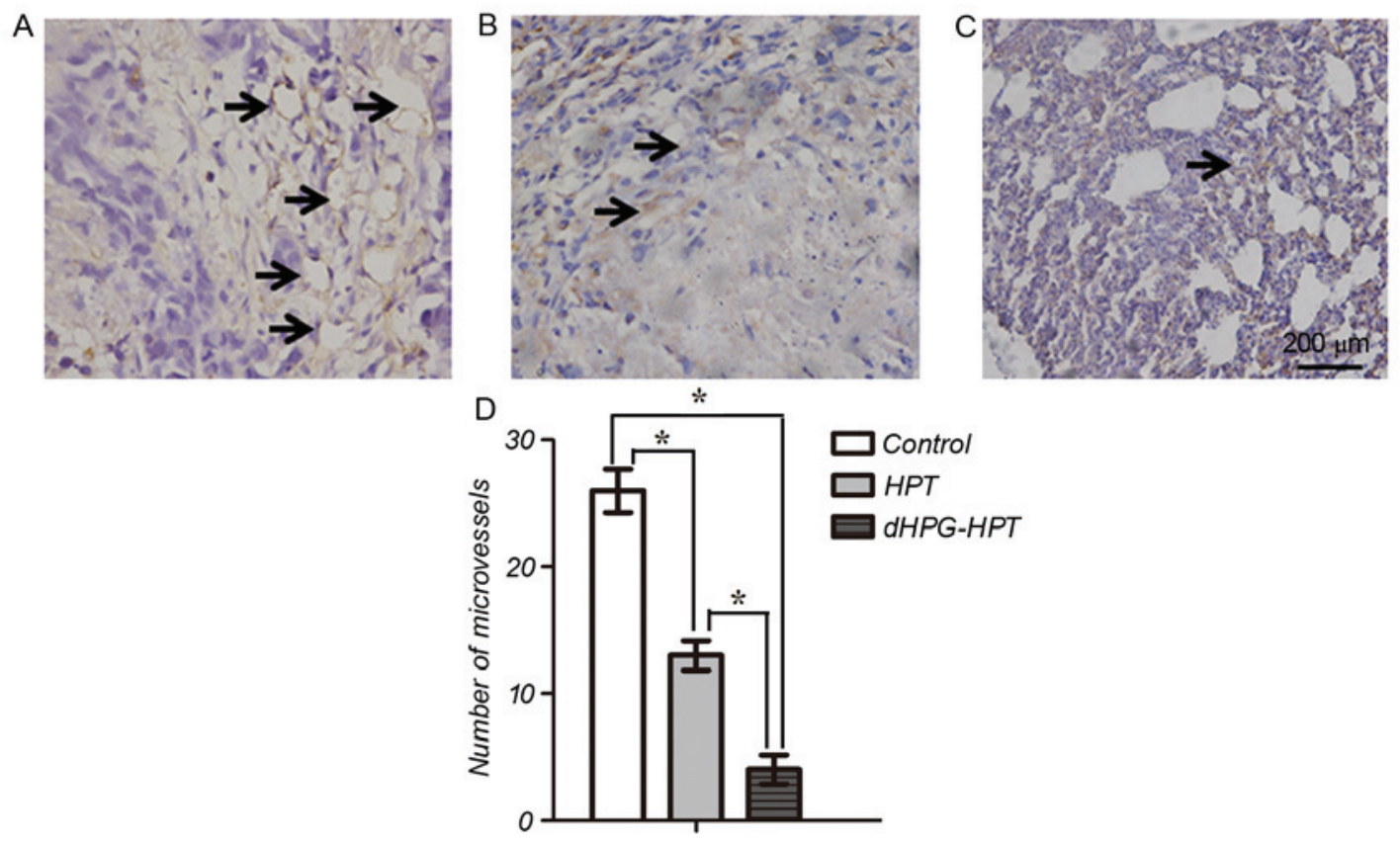

Figure 4. The expression of CD34 in the tissue samples of (A) the control group, (B) HPT group and (C) dHPG-HPT group. (D) Shows the number of microvessels in each group. The arrows indicate microvessels. Scale bar, $100 \mu \mathrm{m} .{ }^{*} \mathrm{P}<0.05$.

in tumors of the HPT and control groups (dHPG-HPT: 5 \pm 1.15 ; HPT: $29 \pm 1.74$; control: $60 \pm 2.53$ ) (Fig. 5D). This result suggested that dHPG-HPT may be more efficient in the inhibition of SACC cell proliferation.

dHPG-HPT may inhibit the invasion capacity of SACC cells. Matrix metalloproteinase 9 (MMP9) plays a critical role in tumor invasion and metastasis (10). Immunohistochemistry (Fig. 6) showed that the expression of MMP9 in tumors of the dHPG-HPT group was dramatically decreased compared with that in tumors of the HPT group (IOD (sum)/area (sum): dHPG-HPT, 0.005 vs. HPT, 0.03; P<0.01) (Fig. 6D). This result suggested that dHPG-HPT may be more efficient in the inhibition of the invasion and metastasis of SACC cells.

\section{Discussion}

As a drug carrier, dHPG has many advantages. First, this compound has a large number of terminal functional groups, which can be used to conjugate drugs and fluorescent groups. 

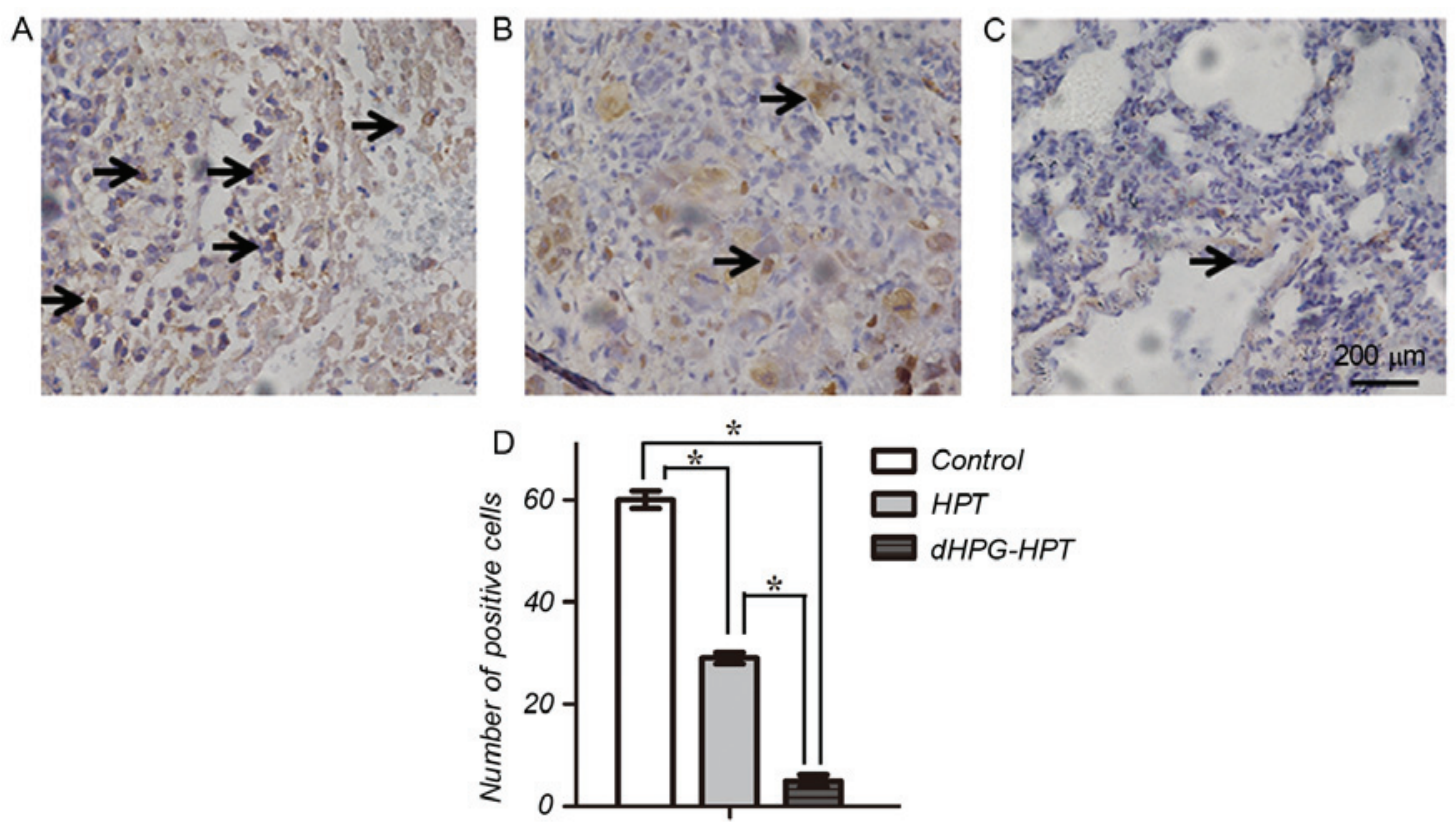

Figure 5. The expression of PCNA in the tissue samples of (A) the control group, (B) HPT group and (C) dHPG-HPT group. (D) Shows the number of positive cells in each group. The arrows indicate positive cells. Scale bars, $100 \mu \mathrm{m}$. ${ }^{*} \mathrm{P}<0.05$.

Second, dHPG has good solubility and dissolves in the blood, which carries the drugs to tissues, which indicates limited drug toxicity and side effects. Moreover, as a drug carrier, dHPG can be degraded in vivo, and the resulting non-toxic degradation products can be absorbed and metabolized. Furthermore, dHPG can target tumor tissues through the 'enhanced permeability and retention' (EPR) effect and release the drugs only in the targeted tissues. Thus, gross observation revealed that the number of SACC metastatic nodules in the lungs was significantly decreased in the dHPG-HPT group compared with the HPT group, suggesting that compared with HPT, dHPG-HPT was more capable of inhibiting the growth of SACC lung metastasis in vivo.

The passive accumulation of macromolecules $(>20 \mathrm{kDa})$ in solid tumors is a phenomenon that might be a potential biological target for tumor-selective drug delivery. The rationale for the use of macromolecules as efficient carriers for the delivery of antitumor agents, even if these molecules are not targeted to an antigen or receptor on the surface of the tumor cell, is based on the work of Matsumura and Maeda (11). Differences in the biochemical and physiological characteristics of normal and malignant tissues are responsible for the passive accumulation of macromolecules in tumors. This feature, termed the EPR effect $(12,13)$, is schematically depicted in Fig. 7. Briefly, low-molecular-weight compounds can easily diffuse into normal and tumor tissues through the endothelial cell layer of blood capillaries. However, macromolecules cannot pass through the capillary walls of normal tissues. Macromolecules enter tumor tissues through the capillaries where blood flow is diminished. In contrast to most normal tissues, tumor tissues are rich in new capillaries, and the endothelial layer of the capillaries is fenestrated and leaky; thus, macromolecules and other nanoparticles can reach the malignant tissue. Tumor tissue generally has a defective lymphatic drainage system, resulting in the retention and subsequent accumulation of macromolecules in solid tumors. Due to the EPR effect, compared with HPT, dHPG-HPT can passively accumulate in SACC xenografts and is sustained for a long period of time, which significantly reduces the volume of lung metastases in nude mice and confers a better treatment effect.

VEGF also plays an important role in angiogenesis (14-16) through the promotion of chemotaxis and mitosis of vascular endothelial cells via the targeting of the specific receptors VEGFR-1 and VEGFR-2, which promotes the growth of tumor tissues. In addition, VEGF enhances the vascular permeability of normal blood vessels and tumor vessels $(17,18)$. Thus, VEGF is one of the critical factors that has an impact on the EPR effect. In the present study, the expression of VEGF in the control group was high, indicating that SACC xenografts were in a rapid growth phase; this potentially provides a good condition for the EPR effect. In the dHPG-HPT group, the expression of VEGF was low, and the tumor volumes were smaller than in the HPT group (Fig. 3), which suggests that the growth of SACC xenografts was inhibited in the dHPG-HPT group. Due to the EPR effect, dHPG-HPT has a passive targeting effect on tumor tissue and a better inhibitory effect on the growth of tumor tissue; thus, the expression of VEGF in the dHPG-HPT group was reduced.

In 1992, Weidner et al (9) reported on MVD, which was used to quantify the amount of new blood vessels in tumors. CD34 is typically overexpressed in proliferating vascular endothelial cells and vascular endothelial cells in tumorous vascular tissues and is typically weakly expressed or absent in normal mature vascular endothelial cells. In Fig. 4A, a large number of new CD34-positive microvessels was observed, and the MVD of the control group was high, suggesting that the tumor was rich in microvascular tissue. However, the MVD of the dHPG-HPT and HPT groups was less than that of the control group, suggesting that both HPT and dHPG-HPT 

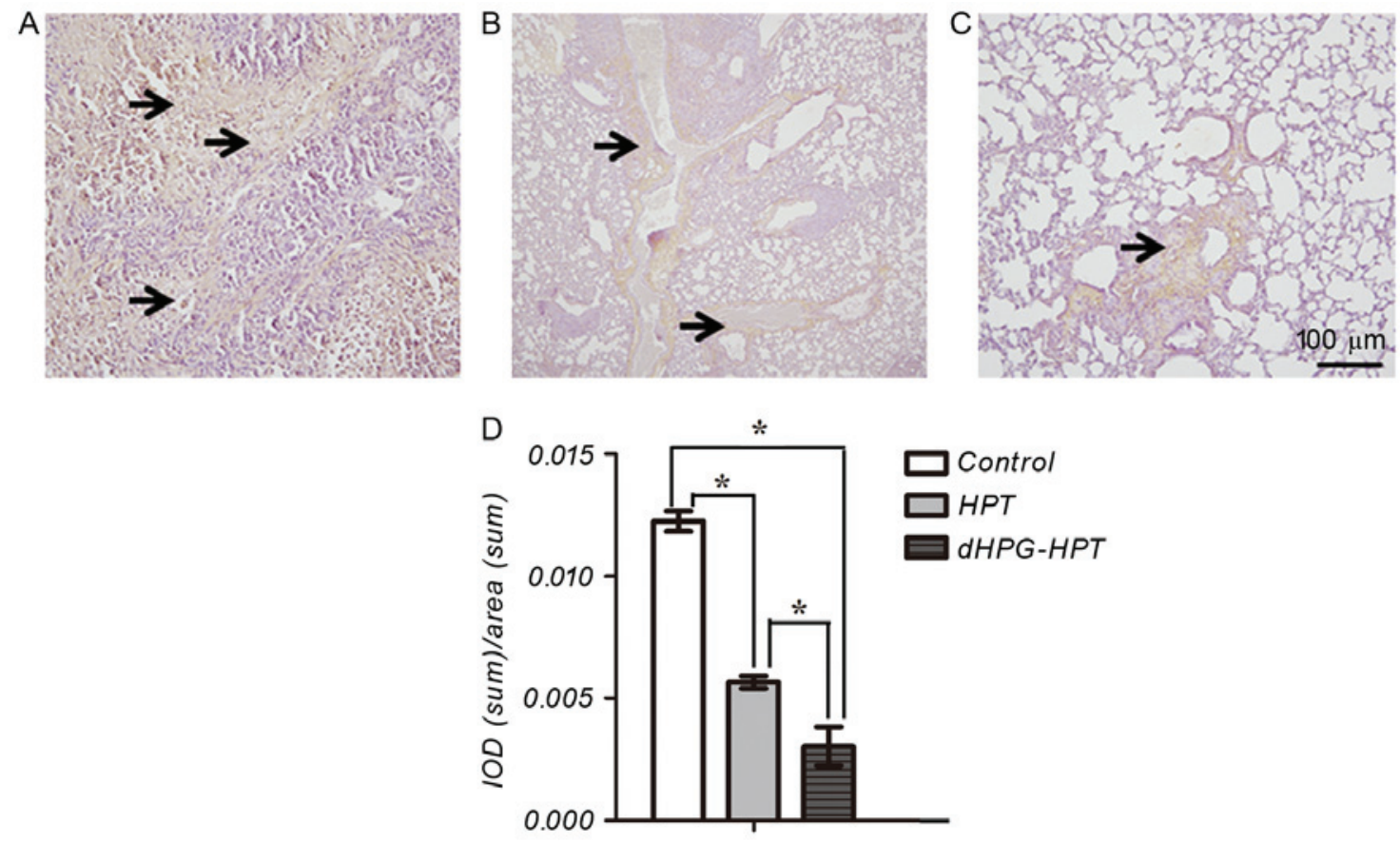

Figure 6. The expression of VEGF in the tissue samples of (A) the control group, (B) HPT group and (C) dHPG-HPT group. (D) Shows the IOD (sum)/area (sum) of each group. The arrows indicate the positive signal. Scale bar, $100 \mu \mathrm{m}$. "P<0.05.

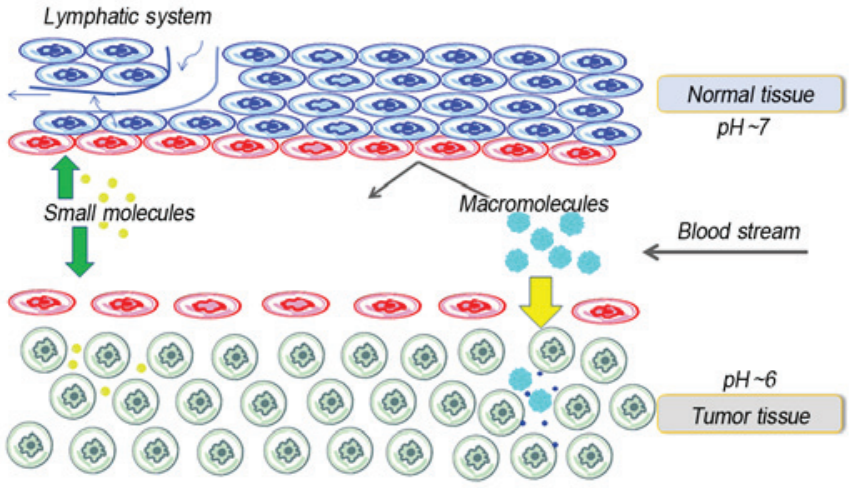

Figure 7. Schematic representation of the anatomical and physiological characteristics of normal and tumor tissue with respect to the EPR effect.

exerted an inhibitory effect on pulmonary metastases of SACC. Since dHPG-HPT utilizes the EPR effect for a passive targeting effect, which can largely inhibit the formation of tumor tissue, the amount of neovascularization in tumor tissue and the MVD of tumor tissue is reduced, and therefore, the MVD was lowest in the dHPG-HPT group.

PCNA is an auxiliary component of DNA polymerase delta and is necessary for DNA replication. PCNA is synthesized during G1 phase of the cell cycle and its expression peaks during $\mathrm{S}$ phase, during which it plays an important role in the regulation of DNA replication. The synthesis and expression of PCNA is closely related to the cell proliferation status and is a major biological indicator that reflects the proliferation of cells $(19,20)$. In the control group, PCNA-positive cells were detected in the tumor tissue, and the number of positive cells was significantly higher than that in the other two groups (Fig. 5), indicating that the SACC-derived tumors were in the proliferation phase. In contrast, the number of PCNA-positive cells was lower in the HPT group, as HPT exerted an inhibitory effect on the growth of SACC. The number of PCNA-positive cells was lowest in the dHPG-HPT group, indicating that dHPG-HPT showed the strongest inhibition of the proliferation of SACC.

A prerequisite for tumor invasion and metastasis is the destruction of the integrity of the basement membrane (21), which occurs during the interaction between tumor cells and the extracellular matrix. Matrix metalloproteinases (MMPs), which degrade extracellular matrix components, play a critical role in tissue remodeling during the development of pathological processes, including inflammation, tissue repair, tumor invasion, and metastasis $(10,22)$. Among MMPs, MMP9 is of particular interest because this protein, secreted in the form of a zymogen, is activated to form collagen IV and V as well as gelatin structures, such as the basement membrane and connective tissue. MMP9 plays an important role in tumor metastasis $(23,24)$. SACC has a strong capacity for invasion, easily invades local normal tissue and metastasizes to distal organs. Thus, the expression of MMP9 in the control group was highest (Fig. 6). However, the expression of MMP9 in the HPT group was decreased and was lowest in the dHPG-HPT group, implying that both HPT and dHPG-HPT reduce the invasiveness and metastatic capability of SACC; between these two, dHPG-HPT led to a greater reduction.

dHPG-HPT can effectively reduce the expression of VEGF, CD34, PCNA and MMP9 in SACC xenografts and suppress angiogenesis, proliferation and invasion of SACC cells in vivo, indicating a good therapeutic effect on SACC xenografts in nude mice. This study provides a theoretical basis for the application of polymeric drug carriers in the treatment of SACC. 


\section{Acknowledgments}

The present study was supported by the Shanghai Science and Technology Commission (no. 12nm0503002).

\section{References}

1. Bell D and Hanna EY: Salivary gland cancers: Biology and molecular targets for therapy. Curr Oncol Rep 14: 166-174, 2012

2. Rapidis AD, Givalos N, Gakiopoulou H, Faratzis G, Stavrianos SD Vilos GA, Douzinas EE and Patsouris E: Adenoid cystic carcinoma of the head and neck. Clinicopathological analysis of 23 patients and review of the literature. Oral Oncol 41: 328-335, 2005.

3. van der Wal JE, Becking AG, Snow GB and van der Waal I: Distant metastases of adenoid cystic carcinoma of the salivary glands and the value of diagnostic examinations during follow-up. Head Neck 24: 779-783, 2002

4. Kokemueller H, Eckardt A, Brachvogel P and Hausamen JE: Adenoid cystic carcinoma of the head and neck-a 20 years experience. Int J Oral Maxillofac Surg 33: 25-31, 2004.

5. Liu Y, Lou C, Yang H, Shi M and Miyoshi H: Silica nanoparticles as promising drug/gene delivery carriers and fluorescent nano-probes: Recent advances. Curr Cancer Drug Targets 11: 156-163, 2011

6. Mourelatou EA, Libster D, Nir I, Hatziantoniou S, Aserin A Garti N and Demetzos C: Type and location of interaction between hyperbranched polymers and liposomes. Relevance to design of a potentially advanced drug delivery nanosystem (aDDnS). J Phys Chem B 115: 3400-3408, 2011.

7. Xu Q, Liu Y, Su S, Li W, Chen C and Wu Y: Anti-tumor activity of paclitaxel through dual-targeting carrier of cyclic RGD and transferrin conjugated hyperbranched copolymer nanoparticles. Biomaterials 33: 1627-1639, 2012.

8. Hu M, Chen M, Li G, Pang Y, Wang D, Wu J, Qiu F, Zhu X and Sun J: Biodegradable hyperbranched polyglycerol with ester linkages for drug delivery. Biomacromolecules 13: 3552-3561, 2012.

9. Weidner N, Folkman J, Pozza F, Bevilacqua P, Allred EN, Moore DH, Meli S and Gasparini G: Tumor angiogenesis: A new significant and independent prognostic indicator in early-stage breast carcinoma. J Natl Cancer Inst 84: 1875-1887, 1992.

10. Egeblad M and Werb Z: New functions for the matrix metalloproteinases in cancer progression. Nat Rev Cancer 2: 161-174, 2002.

11. Matsumura $\mathrm{Y}$ and Maeda $\mathrm{H}$ : A new concept for macromolecular therapeutics in cancer chemotherapy: Mechanism of tumoritropic accumulation of proteins and the antitumor agent smancs. Cancer Res 46: 6387-6392, 1986.
12. Maeda H, Wu J, Sawa T, Matsumura Y and Hori K: Tumor vascular permeability and the EPR effect in macromolecular therapeutics: A review. J Control Release 65: 271-284, 2000.

13. Maeda $\mathrm{H}$ and Matsumura Y: Tumoritropic and lymphotropic principles of macromolecular drugs. Crit Rev Ther Drug Carrier Syst 6: 193-210, 1989.

14. Senger DR, Galli SJ, Dvorak AM, Perruzzi CA, Harvey VS and Dvorak HF: Tumor cells secrete a vascular permeability factor that promotes accumulation of ascites fluid. Science 219: 983-985, 1983.

15. Rosenthal RA, Megyesi JF, Henzel WJ, Ferrara N and Folkman J: Conditioned medium from mouse sarcoma 180 cells contains vascular endothelial growth factor. Growth Factors 4: 53-59, 1990.

16. Abhinand CS, Raju R, Soumya SJ, Arya PS and Sudhakaran PR: VEGF-A/VEGFR2 signaling network in endothelial cells relevant to angiogenesis. J Cell Commun Signal 10: 347-354, 2016.

17. Maeda H, Fang J, Inutsuka $\mathrm{T}$ and Kitamoto $\mathrm{Y}$ : Vascular permeability enhancement in solid tumor: Various factors, mechanisms involved and its implications. Int Immunopharmacol 3: 319-328, 2003.

18. Maeda $\mathrm{H}$ : The enhanced permeability and retention (EPR) effect in tumor vasculature: The key role of tumor-selective macromolecular drug targeting. Adv Enzyme Regul 41: 189-207, 2001.

19. Maga $G$ and Hubscher U: Proliferating cell nuclear antigen (PCNA): A dancer with many partners. J Cell Sci 116: 3051-3060, 2003.

20. Hall PA, Levison DA, Woods AL, Yu CC, Kellock DB Watkins JA, Barnes DM, Gillett CE, Camplejohn R, Dover R, et al: Proliferating cell nuclear antigen (PCNA) immunolocalization in paraffin sections: An index of cell proliferation with evidence of deregulated expression in some, neoplasms. J Pathol 162: 285-294, 1990

21. Safina AF,VendetteE, Varga A andBakin AV:TGF-beta-p38MAPK signaling contributes to tumor invasion and pulmonary metastases by increasing MMP9 activity and cell motility. Cancer Res 65: 2005.

22. Stetler-Stevenson WG: Matrix metalloproteinases in angiogenesis: A moving target for therapeutic intervention. J Clin Invest 103: 1237-1241, 1999.

23. Soechting JF and Flanders M: Sensorimotor representations for pointing to targets in three-dimensional space. J Neurophysiol 62 : $582-594,1989$.

24. Belotti D, Paganoni P, Manenti L, Garofalo A, Marchini S, Taraboletti G and Giavazzi R: Matrix metalloproteinases (MMP9 and MMP2) induce the release of vascular endothelial growth factor (VEGF) by ovarian carcinoma cells: Implications for ascites formation. Cancer Res 63: 5224-5229, 2003. 